\title{
Evaluación Mecánica y Ambiental del Uso de Ceniza Volante con Activación Alcalina como Alternativa de Reemplazo Total del Cemento en la Elaboración de Tabletas Prefabricadas
}

Laura C. Prieto(1)* ${ }^{(1)}$ Arés A. Montaño(1), Alejandro Parra(1), y Julián D. Puerto-Suárez ${ }^{(2)}$

(1) Universidad de La Salle, Facultad de Ingeniería, Programa de Ingeniería Ambiental y Sanitaria, Carrera 2 No. 10 70, Bogotá D.C.-Colombia. (e-mail: lauraprieto35@unisalle.edu.co; mandres46@unisalle.edu.co; alparra@unisalle.edu.co).

(2) Universidad Nacional de Colombia, Facultad de Ingeniería, Departamento de Ingeniería Civil y Agrícola, Carrera 45 No. 26 - 85, Bogotá D.C.-Colombia. (e-mail: jdpuertos@unal.edu.co).

* Autor a quien debe ser enviada la correspondencia

Recibido Ago. 20, 2018; Aceptado Nov. 6, 2018; Versión final Ene. 3, 2019, Publicado Jun. 2019

\section{Resumen}

La presente investigación está orientada a la implementación de ceniza volante activada alcalinamente como sustitución total del cemento Portland en la elaboración de tabletas prefabricadas. El programa experimental comprendió la caracterización fisicoquímica de materias primas, hallazgo de la relación óptima activador alcalino/ceniza volante y ensayos mecánicos de resistencia a la compresión y módulo de rotura. Adicionalmente se incluyó un comparativo de la huella de carbono de un lote de tabletas prefabricadas de mortero de cemento Portland y ceniza volante. La activación de ceniza volante se realizó por medio de la combinación de activadores alcalinos compuestos por $\mathrm{NaOH}$ y $\mathrm{Na}_{2} \mathrm{SiO}_{3}$. Los resultados demostraron que los morteros de ceniza volante presentan buenas propiedades mecánicas y viabilidad ambiental en términos de huella de carbono. La ceniza volante puede ser acogida en la industria de tabletas prefabricadas como opción de menor impacto ambiental con óptimas resistencias a la compresión y módulo de rotura.

Palabras clave: ceniza volante; cemento Portland; tableta prefabricada; activador alcalino; huella de carbono

\section{Mechanical and Environmental Assessment of the Use of Fly Ash with Alkaline Activation as an Alternative to Total Replacement of Cement in the Manufacture of Prefabricated Tablets}

\begin{abstract}
The present investigation is focused on the implementation of alkaline activated fly ash as a total replacement of Portland cement in the manufacture of prefabricated tablets. The experimental program included the physico-chemical characterization of raw materials, finding the optimum alkaline activator/fly ash ratio and mechanical tests of compressive strength and rupture modulus. Additionally, a comparison of the carbon footprint of a batch of prefabricated tablets made from Portland cement mortar and fly ash was also performed. The activation of the fly ash was carried out by means of the combination of alkaline activators composed of $\mathrm{NaOH}$ and $\mathrm{Na}_{2} \mathrm{SiO}_{3}$. The results obtained showed that fly ash mortars have good mechanical properties and environmental viability in terms of carbon footprint. The fly ash can be used in the industry of prefabricated tablets as an option of lower environmental impact with optimal resistance to compression and rupture modulus.
\end{abstract}




\section{INTRODUCCIÓN}

La producción de cemento Portland a nivel global ha significado la extracción, transformación y disminución de recursos naturales y ha promovido un aumento en impactos ambientales significativos en cada una de sus etapas de fabricación, generando la liberación de aproximadamente $0,85 \mathrm{Kg}$ de $\mathrm{CO} 2$ a la atmósfera por cada kilogramo de cemento procesado representando 5-7\% de las emisiones globales de CO2 (Khan et al., 2016), adicionalmente se requieren $3400 \mathrm{MJ} / T o n$ para su elaboración que corresponden a 2,50 \% de la energía mundial utilizada (Hoyos-Montilla et al., 2018). En Colombia la producción del cemento Portland destinada a prefabricados es de aproximadamente 280,52 Ton/año (Dane, 2018a), representando un papel fundamental como materia prima para el desarrollo y crecimiento de la economía colombiana aportando cerca del $7,28 \%$ al PIB del país (Dane, 2018b).

El aporte más relevante de emisiones de $\mathrm{CO} 2$ por la industria del cemento es debido a la descarbonatación de la piedra caliza por exposición a elevadas temperaturas generando $\mathrm{CO} 2$ y $\mathrm{CaO}$ (Sánchez Soloaga et al., 2012), por tanto se debe tener como prioridad la sustitución del uso de caliza para aliviar parcialmente tal situación y utilizar materiales alternativos cementosos de reemplazo parcial o total del cemento Portland tales como los geopolímeros. En la implementación de dichos materiales se utiliza ceniza volante, subproducto de la quema de carbón pulverizado en centrales termoeléctricas expuesto a temperaturas entre 1200 y $1700 \stackrel{\circ}{ } \mathrm{C}$, compuesto principalmente por minerales no combustibles en forma de finas cenosferas retenidas por filtros que evitan su emisión a la atmósfera (Mejía et al., 2014). La producción de ceniza volante a nivel global es significativa dado que el $37 \%$ de la electricidad mundial se obtiene con carbón (World Coal Association, 2018), por el contrario en Colombia la generación de energía eléctrica se concentra principalmente por hidroeléctricas con una participación del $63,90 \%$, seguidas de termoeléctricas a base de gas natural con $26,30 \%$ y finalmente se encuentran las termoeléctricas a base de carbón abarcando $4,80 \%$, las cuales producen aproximadamente $600 \mathrm{KTon} / \mathrm{año}$ de ceniza volante de calidad variable, en su gran mayoría no se está aprovechando adecuadamente y se constituye en un problema ambiental (Upme, 2015).

Por otro lado, investigaciones recientes han demostrado que para la geopolimerización de ceniza volante el tamaño máximo de partícula debe ser de aproximadamente 150-250 $\mu \mathrm{m}$, contemplando una morfología esférica y estructura amorfa con alto contenido de sílice y óxido de aluminio (Rieger et al., 2017) . Estos son liberados durante el proceso de geopolimerización debido al ambiente altamente alcalino proporcionado por las soluciones activadoras las cuales son álcalis solubles que generalmente están compuestas de sodio y/o potasio (Azevedo y Strecker, 2017). La solución alcalina controla la división de los enlaces Si-O y Al-O produciendo el endurecimiento del geopolímero por medio del mecanismo de policondensación (Rieger et al., 2017). El tipo y nivel de concentración del activador influyen significativamente en las propiedades mecánicas y microestructurales de los geopolímeros (Yousefi Oderji et al., 2017). En consecuencia la utilización de un activador compuesto de solución de hidróxido de sodio $(\mathrm{NaOH})$ y silicato de sodio (Na2SiO3) proporciona mejores propiedades mecánicas que el uso único de la solución activadora de $\mathrm{NaOH}$ (Lăzărescu et al., 2017). La adición del $\mathrm{Na} 2 \mathrm{SiO} 3$ a la solución de $\mathrm{NaOH}$ incrementa la resistencia del geopolímero aumentando las relaciones $\mathrm{SiO} 2$ / Al2O3 y SiO2 / Na2O que proporcionan finalmente la formación de más enlaces Si-O-Si en el producto final (Bhagath Singh y Subramaniam, 2017a).

Los parámetros más influyentes en la producción de geopolímeros de ceniza volante activada alcalinamente son, concentración de $\mathrm{NaOH}$, temperatura de curado, edad de curado, relación de la solución activadora (Na2SiO3 / NaOH) y proporción activador alcalino / ceniza volante (AA/CV). Lăzărescu et al., (2017) en su investigación sugieren proporciones de solución de $\mathrm{Na2SiO3/NaOH}$ en el rango de 0,40 a 2,50 y relaciones (AA/CV) entre 0,50 y 3. Para evitar resultados adversos de la resistencia del material geopolimérico se debe tener en cuenta la concentración de $\mathrm{NaOH}$ en un rango óptimo de 8 a 16 M (Saloma et al., 2016; Lăzărescu et al., 2017). Según Hoyos-Montilla et al., (2018) cuando la molaridad de $\mathrm{NaOH}$ es baja se forman pocos núcleos y el flujo máximo de calor disminuye por tanto el aumento de la cantidad del $\mathrm{NaOH}$ es directamente proporcional al flujo máximo de calor. La temperatura de curado es un precursor para que la reacción de geopolimerización se obtenga en un tiempo óptimo. A temperatura ambiente el proceso de disolución de la ceniza volante en una solución alcalina no ocurre inmediatamente debido a la barrera energética de activación generando que la pasta tarde en endurecer (Chithiraputhiran y Neithalath, 2013). Por el contrario al aumentar la temperatura ocurre policondensación, proceso de formación de geles que proporcionan la resistencia mecánica (Chithiraputhiran y Neithalath, 2013). Según estudios previos el rango óptimo de curado es de $60^{\circ} \mathrm{C}$ a $85^{\circ} \mathrm{C}$ (Bhagath Singh y Subramaniam, 2017b). Sin embargo, no se descarta que a temperatura ambiente se logre dicha reacción.

Teniendo en cuenta la importancia que tiene la implementación de materiales sostenibles en la sustitución del cemento Portland para la disminución de emisiones de CO2-eq a la atmósfera, se ejecutan medidas de reducción y/o compensación de emisiones. El cálculo de la huella de carbono se convierte en un indicador para determinar impactos y su posterior disminución y así cuantificar la cantidad de emisiones de gases de 
efecto invernadero (GEI) asociadas al ciclo de vida de un producto, para determinar su contribución al cambio climático (Espíndola y Valderrama, 2012). Debido al interés de la población mundial frente a esta problemática ambiental se han creado metodologías para el cálculo de la huella de carbono. En el reporte de la Comisión Europea se resaltan cuatro procedimientos principales para la estimación de la huella de carbono en empresas, organizaciones, servicios, procesos y productos. Los cuales son, protocolo de gases efecto invernadero, balance de carbono, PAS 2050 y método compuesto de las cuentas contables (European Comission DG Environment, 2010). Para el cálculo de la huella de carbono de un producto es comúnmente usado el método de las Especificaciones Públicamente Disponibles (Publicly Available Specification), llamado PAS 2050, elaborado en el año 2007 por el Instituto Británico de Estandarización. Está enfocado al cálculo de emisiones de productos y servicios por medio del análisis del ciclo de vida y huella de carbono (Espíndola y Valderrama, 2012; PAS 2050, 2014).

Esta investigación pretende determinar la mezcla geopolimérica óptima que cumpla con los requisitos de los ensayos mecánicos de resistencia a la compresión y módulo de rotura. Esto con el fin de utilizar ceniza volante activada alcalinamente como reemplazo total del cemento Portland en la elaboración de tabletas prefabricadas y así evaluar su viabilidad ambiental en términos de huella de carbono. Cabe resaltar que cualquier ensayo mecánico para tabletas prefabricadas que no se haya mencionado, no hace parte de esta investigación y estará sujeto a futuras investigaciones.

\section{MATERIALES Y MÉTODOS}

Se describen los materiales utilizados involucrando sus características fisicoquímicas, el procedimiento experimental para la creación de las muestras de cemento Portland y ceniza volante y finalmente los métodos de prueba y caracterizaciones realizados.

\section{Materiales utilizados}

La ceniza volante utilizada fue suministrada por la planta termoeléctrica de Termozipa ubicada en Zipaquirá Colombia, la composición química que se muestra en la Tabla 1 se determinó por medio del espectrómetro de fluorescencia de rayos $\mathrm{X}$ (XRF). Los componentes de óxidos de sílice, aluminio y hierro son aproximadamente de $84 \%$ cumpliendo con lo establecido en la (ASTM C618-12, 2014), (>70\%) para ceniza volante tipo $\mathrm{F}$. Se realizaron las pruebas físicas de densidad y finura según lo estipulado por la (ASTM C311, 2015) arrojando valores de $2229 \mathrm{Kg} / \mathrm{m}_{3}$ y $31 \%$ respectivamente, indicando que la ceniza volante utilizada es óptima para ser activada alcalinamente.

Tabla 1: Composición química de ceniza volante. * LOI pérdida por ignición a 950 ㄷ.

\begin{tabular}{llllllllllll}
\hline $\mathrm{SiO}_{2}$ & $\mathrm{Al}_{2} \mathrm{O}_{3}$ & $\mathrm{Fe}_{2} \mathrm{O}_{3}$ & $\mathrm{CaO}$ & $\mathrm{Na}_{2} \mathrm{O}$ & $\mathrm{K}_{2} \mathrm{O}$ & $\mathrm{MgO}$ & $\mathrm{MnO}$ & $\mathrm{TiO}_{2}$ & $\mathrm{SO}_{3}$ & $\mathrm{P}_{2} \mathrm{O}_{5}$ & LOI $^{*}$ \\
\hline 50,38 & 24,14 & 9,50 & 5,60 & 0,59 & 2,15 & 1,93 & 0,09 & 1,04 & 0,48 & 0,58 & 3,30 \\
\hline
\end{tabular}

La solución alcalina se elaboró mezclando a diferentes proporciones solución de silicato de sodio $\left(\mathrm{Na}_{2} \mathrm{SiO}_{3}\right)$, compuesto de $28,18 \% \mathrm{SiO}_{2}, 8,62 \%, \mathrm{Na}_{2} \mathrm{O}$ y $\mathrm{H}_{2} \mathrm{O} 63,20 \%$ con hidróxido de sodio $(\mathrm{NaOH})$ al $98,50 \%$ de pureza a diferentes molaridades. La preparación del hidróxido de sodio se realizó disolviendo gránulos de $\mathrm{NaOH}$ en un litro de agua desionizada dependiendo la molaridad seleccionada. La solución se dejó reposar por $24 \mathrm{~h}$ antes de su utilización. Se utilizó cemento Portland tipo I (ASTM C150, 2014) según información entregada por el fabricante Cemex, tiene superficie específica Blaine de $3000 \mathrm{~cm}^{2} / \mathrm{gr}$ y resistencia mínima a la compresión a 28 días de $24 \mathrm{MPa}$, su densidad calculada fue de $3100 \mathrm{~kg} / \mathrm{m}^{3}$. La arena de río fue suministrada por el depósito de materiales la concordia Ltda. Se realizaron pruebas de caracterización densidad, humedad total y porcentaje de arcilla, las cuales obtuvieron como resultado $2394 \mathrm{Kg} / \mathrm{m}^{3}, 8,93 \%$ y $2,23 \%$ respectivamente. La granulometría se muestra en la Fig. 1 donde se compararon los resultados obtenidos con los rangos mínimos y máximos establecidos en la (ASTM C33, 2018).

\section{Procedimiento experimental}

Los morteros de cemento Portland se prepararon a una relación cemento/arena de 1:2,75 según (ASTM C109M, 2016). La relación agua/cemento (A/C) fue determinada por la prueba de fluidez arrojando un valor A/C 0,48. La mezcla se realizó en un mezclador Humboldt H-3853.3F. Primero se mezcló el cemento y la arena durante 1 minuto a una velocidad de $(285 \pm 10 \mathrm{rpm})$, luego se agregó el agua y se mezcló por 5 minutos a la misma velocidad. Los morteros se vertieron en moldes de $0,50 \mathrm{~m}^{3}$, estos fueron golpeados 32 veces en dos capas para eliminar el aire atrapado y se dejaron fraguar durante 24 horas a temperatura ambiente. Los cubos desmoldados se curaron en ambiente húmedo durante 7, 14 y 28 días. 


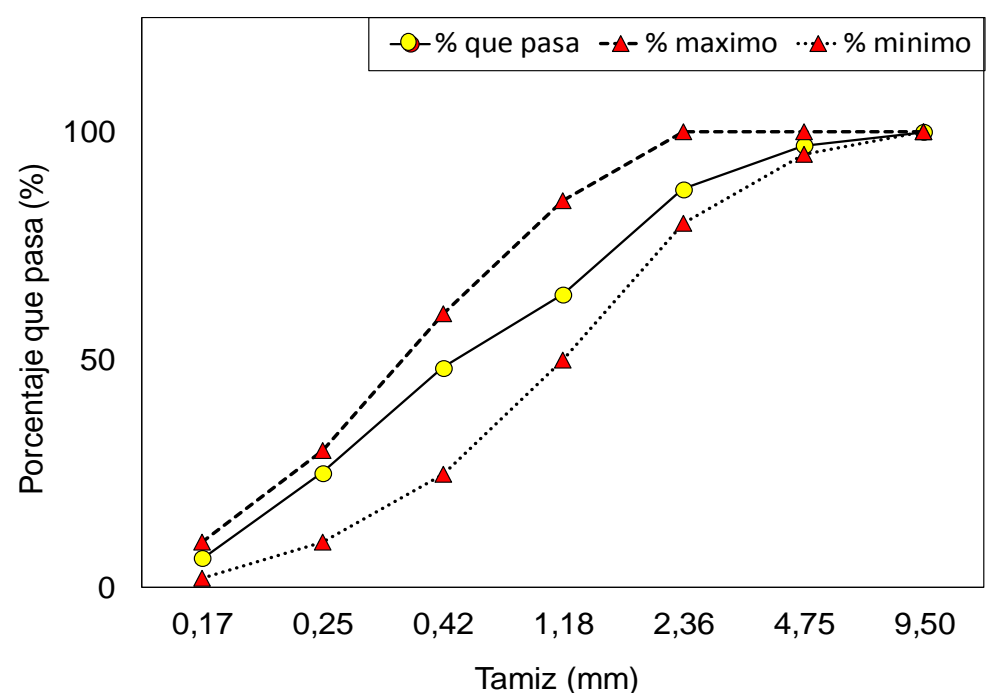

Fig. 1: Granulometría arena de rio.

La fabricación de morteros de ceniza volante activada alcalinamente se realizó por medio de un plan experimental donde se tuvieron en cuenta tres variables, concentraciones de $\mathrm{NaOH}$, relaciones activador alcalino / ceniza volante (AA/CV) y $\mathrm{Na}_{2} \mathrm{SiO}_{3} / \mathrm{NaOH}$. Las concentraciones de $\mathrm{NaOH}$ fueron de 8,10 y $12 \mathrm{M}$ establecidas según Saloma et al., (2016); Lăzărescu et al., (2017), las relaciones AA/CV se determinaron de 0,$40 ; 0,45 ; 0,50 ; 0,60$ y finalmente las proporciones de $\mathrm{Na}_{2} \mathrm{SiO}_{3} / \mathrm{NaOH}$ fueron de $1 ; 1,50 ; 2$ y 2,50. Se realizaron 16 composiciones diferentes por cada una de las concentraciones de $(\mathrm{NaOH})$ tal como se muestra en la Tabla 2. Los tiempos de curado fueron de 24 horas a $80{ }^{\circ} \mathrm{C}$ y 7,14 y 28 días a temperatura ambiente arrojando un total de 576 muestras realizadas. Las 16 proporciones de muestra se mantuvieron para las molaridades de 8,10 y $12 \mathrm{M}$.Durante el curado las muestras se sellaron con plástico con el fin de garantizar condiciones de humedad. La relación ceniza volante/arena fue de 1:2,75 tal como lo especifica la (ASTM C109M, 2016) reemplazando el cemento por ceniza volante.

Tabla 2: Proporción de la mezcla del mortero de geopolímero.

\begin{tabular}{lcc}
\hline Muestra & $\begin{array}{c}\text { Activador alcalino / } \\
\text { ceniza volante }\end{array}$ & $\mathrm{Na}_{2} \mathrm{SiO}_{3} / \mathrm{NaOH}$ \\
\hline G1 & 0,40 & \\
G2 & 0,45 & 1 \\
G3 & 0,50 & \\
G4 & 0,60 & \\
\hline G5 & 0,40 & 1,50 \\
G6 & 0,45 & \\
G7 & 0,50 & \\
G8 & 0,60 & 2 \\
\hline G9 & 0,40 & \\
G10 & 0,45 & \\
G11 & 0,50 & \\
G12 & 0,60 & 2,50 \\
\hline G13 & 0,40 & \\
G14 & 0,45 & \\
G15 & 0,50 & \\
G16 & 0,60 & \\
\hline
\end{tabular}

La nomenclatura de las muestras se clasificó por medio de tres grupos, grupo A (GA) constituye a concentración de $\mathrm{NaOH}$ de $8 \mathrm{M}$, grupo $\mathrm{B}$ (GB) concentración $\mathrm{NaOH} 10 \mathrm{M}$ y grupo $\mathrm{C}$ (GC) concentración $\mathrm{NaOH}$ 12M. Cada grupo fue curado a diferentes edades $24 \mathrm{~h}$ a $80^{\circ} \mathrm{C}, 7,14$ y 28 días a temperatura ambiente. Por 
tanto, a los términos de las muestras se les agregó al final la edad de curado. Ejemplo 1, la muestra GA1-24h corresponde a la mezcla G1 expuesta en la Tabla 2 con concentración $\mathrm{NaOH} 8 \mathrm{M}$ y tiempo de curado de $24 \mathrm{~h}$ a $80^{\circ} \mathrm{C}$. Ejemplo 2, la muestra GB2-7d corresponde a la mezcla G2 de la Tabla 2 con concentración $\mathrm{NaOH}$ $10 \mathrm{M}$ y edad de 7 dias.

La mezcla se realizó pesando ceniza volante, arena y activadores alcalinos. Se vertió la ceniza volante y arena en un mezclador Humboldt $\mathrm{H}-3853.3 \mathrm{~F}$ con capacidad de $0,005 \mathrm{~m}^{3}$, donde se mezcló durante tres

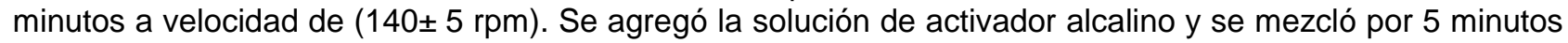
a una velocidad de $(285 \pm 10 \mathrm{rpm})$ hasta que la muestra se homogenizó totalmente. Se depositó la mezcla en cubos de $0,05 \times 0,05 \times 0,05 \mathrm{~m}$ por cada relación establecida por medio de 32 golpes a tres capas para eliminar las burbujas de aire atrapadas y lograr unificar adecuadamente la mezcla. A partir del mortero que obtuvo mayor resistencia a la compresión, se crearon tres tabletas prefabricadas de mortero de ceniza volante activada alcalinamente con los parámetros de diseño según la Norma Técnica Colombiana 4992 (NTC 4992 , 2004).

\section{Métodos de prueba y caracterizaciones}

La determinación de las densidades del cemento y ceniza volante se realizaron de acuerdo a la ASTM C188 utilizando el frasco de Le Chatelier, agregando $64 \mathrm{~g}$ de muestra a la misma temperatura de solución kerosene, se tapó y se giró en círculos horizontales hasta evidenciar que las burbujas de aire hayan desaparecido, se volvió a sumergir en baño de agua a temperatura constante y se tomó la lectura final. La finura de la ceniza volante fue estimada mediante la ASTM C430-96, inicialmente se colocaron $1000 \mathrm{~g}$ de muestra de ceniza volante en el tamiz $45 \mu \mathrm{m}$ (No.325), se lavó la muestra con un chorro suave de agua, se retiró el tamiz de la boquilla rociadora y se ajustó la presión de esta a $(69 \pm 4 \mathrm{kPa})$. Se lavó nuevamente la muestra durante 1 minuto a dicha presión moviendo el tamiz en forma circular a una velocidad de (1 rps) y una distancia de 12 $\mathrm{mm}$ de la boquilla rociadora al tamiz. Se retiró el tamiz de la boquilla rociadora e inmediatamente se enjuagó con agua destilada y se secó en la parte inferior con una toalla húmeda. Finalmente se secó el tamiz a una temperatura aproximada de $55^{\circ} \mathrm{C}$ por una hora, después de enfriado se retiró el residuo del tamiz y se pesó en una balanza analítica.

Para establecer la consistencia del cemento según la ASTM C187, se mezclaron $650 \mathrm{~g}$ de cemento con agua, la mezcla se moldeó en forma de bola de tal manera que se pudo colocar con facilidad en el anillo Vicat. Se dejó descender el émbolo hasta que hizo contacto con la pasta para tomar la lectura inicial. Se aflojó el tornillo fijador de la aguja y se contabilizaron 30 segundos para tomar la lectura final. Se varió la cantidad de agua en la mezcla hasta que la aguja penetró $10 \mathrm{~mm}$ después de 30 segundos, arrojando un valor relación A/C de 0,28. La fluidez del mortero de cemento se realizó en una mesa de flujo donde se colocó un molde de flujo en el centro. El mortero se fabricó según (ASTM C109M, 2016) con relación A/C de 0,48. Se vertió en el molde una capa de mortero de $25 \mathrm{~mm}$ de espesor compactada con 20 golpes. Se llenó el molde en dos capas y se cortó el mortero sobrante hasta lograr una superficie plana. Pasados 60 segundos se levantó el molde del mortero e inmediatamente se dejó caer la mesa 25 veces en 15 segundos desde $13 \mathrm{~mm}$ de altura. Se midieron los diámetros del mortero a lo largo del procedimiento para calcular el diámetro promedio. Se realizó el mismo procedimiento para la fluidez de los diseños de mezcla de los dos morteros de ceniza volante que arrojaron mayor resistencia a la compresión, con el fin de comparar la fluidez de ambos materiales.

El ensayo de microscopía electrónica de barrido (SEM) fue realizado para la ceniza volante en polvo por medio de un microscopio electrónico de barrido (SEM) FEI Quanta 200-R con espectroscopia de rayos X de energía dispersiva (EDS). El procedimiento inició colocando pequeñas cantidades de muestra en una cinta de carbono que se unió a un trozo de acero inoxidable. Se recogieron las imágenes SEM usando un detector de deriva de silicio con un área activa de $2,50 \times 10^{-5} \mathrm{~m}^{2}$ a un voltaje de aceleración de $15000 \mathrm{~V}$ con una distancia de trabajo de $0,02 \mathrm{~m}$ durante el análisis EDS. La prueba de resistencia a la compresión fue determinada de acuerdo la (ASTM $\mathrm{C} 109,2016)$. Se realizó utilizando una máquina de prueba universal United con capacidad de $1000 \mathrm{KN}$ a una velocidad de carga de $1600 \mathrm{~N} / \mathrm{s}$, ancho para ensayo de 0,53 m, profundidad de 0,65 $\mathrm{m}$ y una altura máxima de tensión y compresión de 0,64 m y 0,66 m respectivamente. Los morteros de cemento Portland fueron fallados a los 7,14 y 28 días de curado bajo condiciones de humedad. Los morteros de ceniza volante activada alcalinamente se fallaron 24 horas después de curado a $80^{\circ} \mathrm{C}$ y 7,14 y 28 días a temperatura ambiente. El módulo de rotura se realizó para tabletas prefabricadas de morteros de ceniza volante activada alcalinamente con el fin de cumplir con la (NTC 4992, 2004). El ensayo se hizo por medio de la máquina de prueba universal United según parámetros establecidos en la (ASTM C348-14, 2014). Las tabletas prefabricadas fueron creadas a partir del diseño de mezcla del mortero que dió mayor resistencia a la compresión.

El cálculo de la huella de carbono se determinó por medio del método del ciclo de vida establecido por el (PAS 2050, 2014), para tabletas prefabricadas de mortero de cemento y de mortero de ceniza volante activada alcalinamente asumiendo un lote de $1000 \mathrm{~kg}$ para cada material. 
Inicialmente se realizaron de manera gráfica y esquemática las etapas productivas asociadas al ciclo de vida de un lote de tabletas prefabricadas de mortero de cemento Portland y mortero de ceniza volante activada alcalinamente como se evidencia en las Figs. 2 y 3 respectivamente. El proceso productivo del cemento Portland se determinó realizando una visita a la planta Santa Rosa Cemex ubicada en el municipio de la Calera Cundinamarca con el fin de tener como base un proceso real.

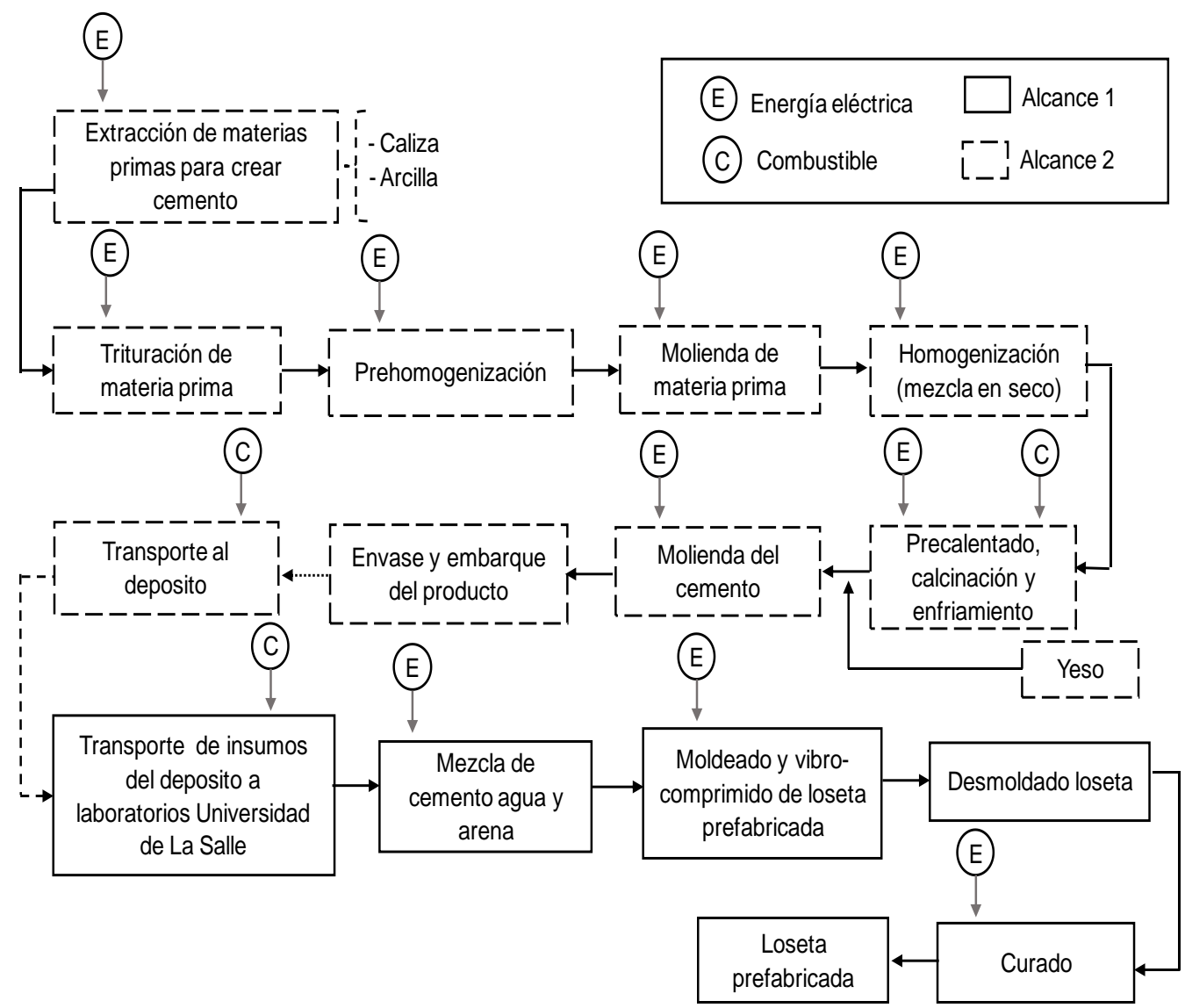

Fig. 2: Proceso productivo para la obtención de tabletas prefabricadas a base de cemento Portland.

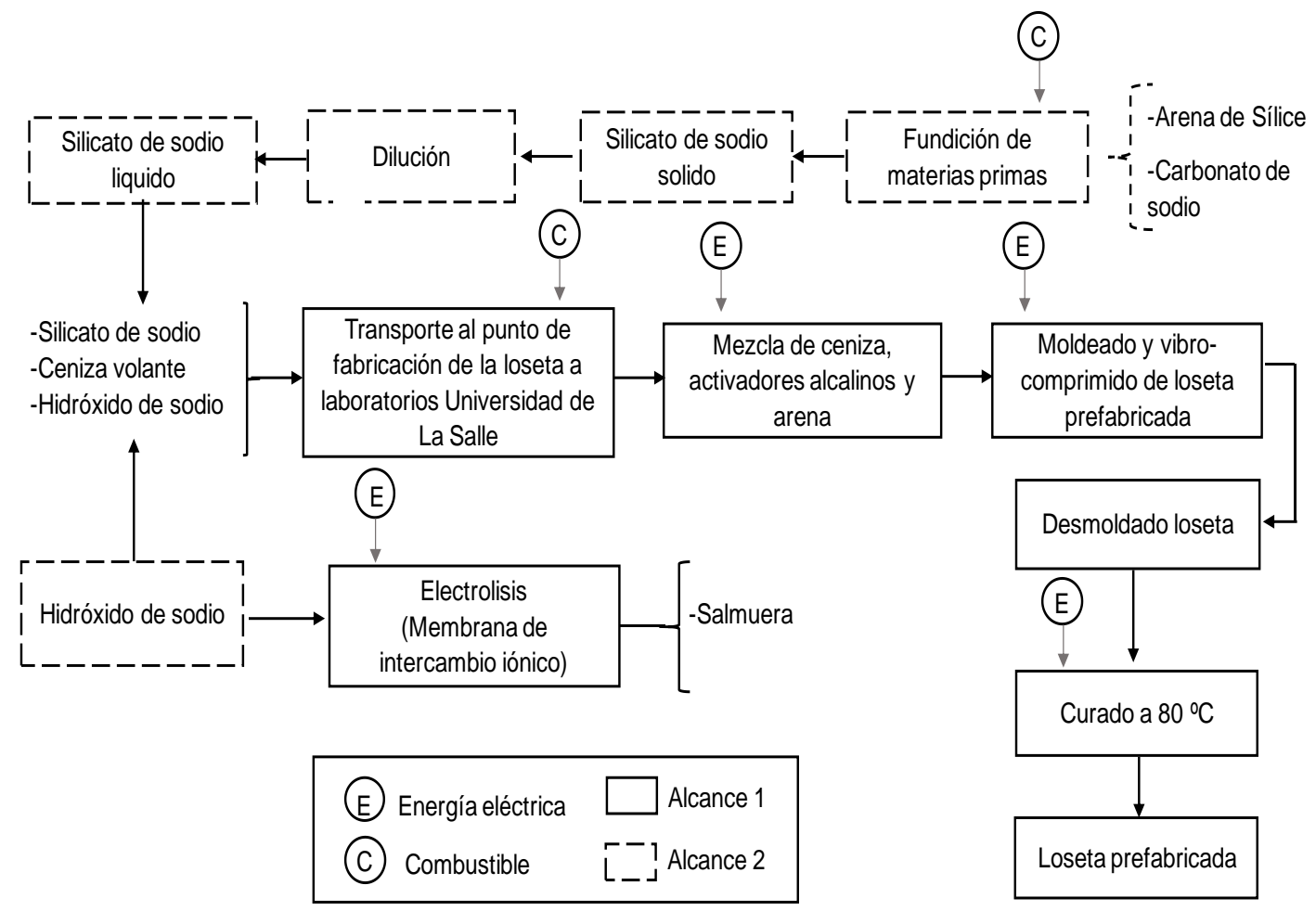

Fig. 3: Proceso productivo para la obtención de tabletas prefabricadas a base de ceniza volante. 
Los límites del cálculo de la huella de carbono se establecieron a partir del límite B2B (business to business) el cual se enfoca en terminar la estimación de la huella de carbono cuando el producto abandona la empresa en la que se ha fabricado quedando por fuera del estudio el proceso de uso y etapa de fin de vida. Este límite fue escogido según lo indicado en la regla de categoría de producto para el cemento y prefabricados de hormigón, definido por las Normas (UNE-EN 15804, 2012) y (UNE $127757,2016)$.

Se definieron dos alcances según el impacto directo e indirecto sobre la fabricación de las tabletas prefabricadas. En el alcance 1 se involucraron las emisiones directas que ocurren de actividades relacionadas en la creación de tabletas prefabricadas, mientras que en el alcance 2 se tuvieron en cuenta las emisiones indirectas derivadas de su fabricación, pero generadas por otras entidades. A partir de las etapas productivas y los límites establecidos para la elaboración de tabletas prefabricadas de cemento y ceniza volante activada alcalinamente, se realizó la recopilación de datos que involucra las cantidades de material y energía implicadas en el ciclo de vida de cada producto y los factores de emisión de los combustibles utilizados.

La recopilación de datos de las tabletas prefabricadas de mortero de cemento fue basada en la visita a la planta Santa Rosa con el fin de obtener mayor precisión. Para las tabletas prefabricadas de mortero de ceniza volante activada alcalinamente los datos se obtuvieron de forma teórica y del proceso práctico realizado en la presente investigación. Para el cálculo de la huella de carbono se tuvieron en cuenta las etapas productivas descritas anteriormente con el fin de crear un balance de masa e identificar fuentes de energía y combustibles utilizados para la fabricación de un lote equivalente a mil (1000) kilogramos. Los cálculos se realizaron por medio de las ecuaciones 1 y 2 mostradas a continuación.

$\mathrm{CE}=\mathrm{E} \times \mathrm{FE}$

Donde $\mathrm{CE}$ es el $\mathrm{CO}_{2}$ eq por consumo energético $\left(\mathrm{Kg} \mathrm{CO}_{2}\right.$ eq); y $\mathrm{E}$ es la electricidad generada $(\mathrm{Wh})$;

$$
\mathrm{CC}=\mathrm{Q} \times \mathrm{EC} \times \mathrm{GWP}
$$

FE es el factor de emisión ( $\mathrm{Kg} \mathrm{CO} 2$ eq/Wh)

Dónde $\mathrm{CC}$ es el $\mathrm{CO}_{2}$ eq por consumo de combustible ( $\mathrm{Kg} \mathrm{CO}_{2}$ eq); $\mathrm{Q}$ es la cantidad de combustible quemado para llevar una actividad en particular; EC es el contenido de energía de los tipos específicos de combustible utilizados para llevar a cabo una actividad; y GWP es el potencial de calentamiento global total del tipo de combustible específico.

\section{RESULTADOS Y DISCUSIÓN}

Para una mejor comprensión, los resultados obtenidos y sus respectivas discusiones se presentan en tres secciones: Microscopia electrónica de barrido (SEM), propiedades mecánicas y huella de carbono.

\section{Microscopía electrónica de barrido (SEM)}

En las Figs. 4 y 5 se presentan la morfología y composición de la muestra de ceniza volante. Se observan partículas esféricas con diferentes tamaños y partículas irregulares porosas que se pueden asociar con inquemados del carbón (Bautista-Ruiz et al., 2017), los cuales por su contenido de materia orgánica limitan la reacción de geopolimerización (Velásquez Vallejo et al., 2007).

La Fig. 4 muestra los detalles morfológicos donde se aprecian algunas plerosferas con superficie esponjosa y alto contenido de cenosferas, también se observa que existen partículas con zonas brillantes producto de la retrodispersión de electrones. En la Fig. 5 se visualiza a mayor detalle una cenosfera de ceniza volante con predominancia en su composición química de $\mathrm{Si}, \mathrm{Al}, \mathrm{O}$ y Fe, corroborando que la muestra tiene una porción considerable de aluminosilicatos debido a la notable cantidad de cenosferas. Se deduce que la ceniza volante al entrar en un ambiente alcalino puede generar exitosamente enlaces Si-O-Si consecuencia de una apropiada geopolimerización. 


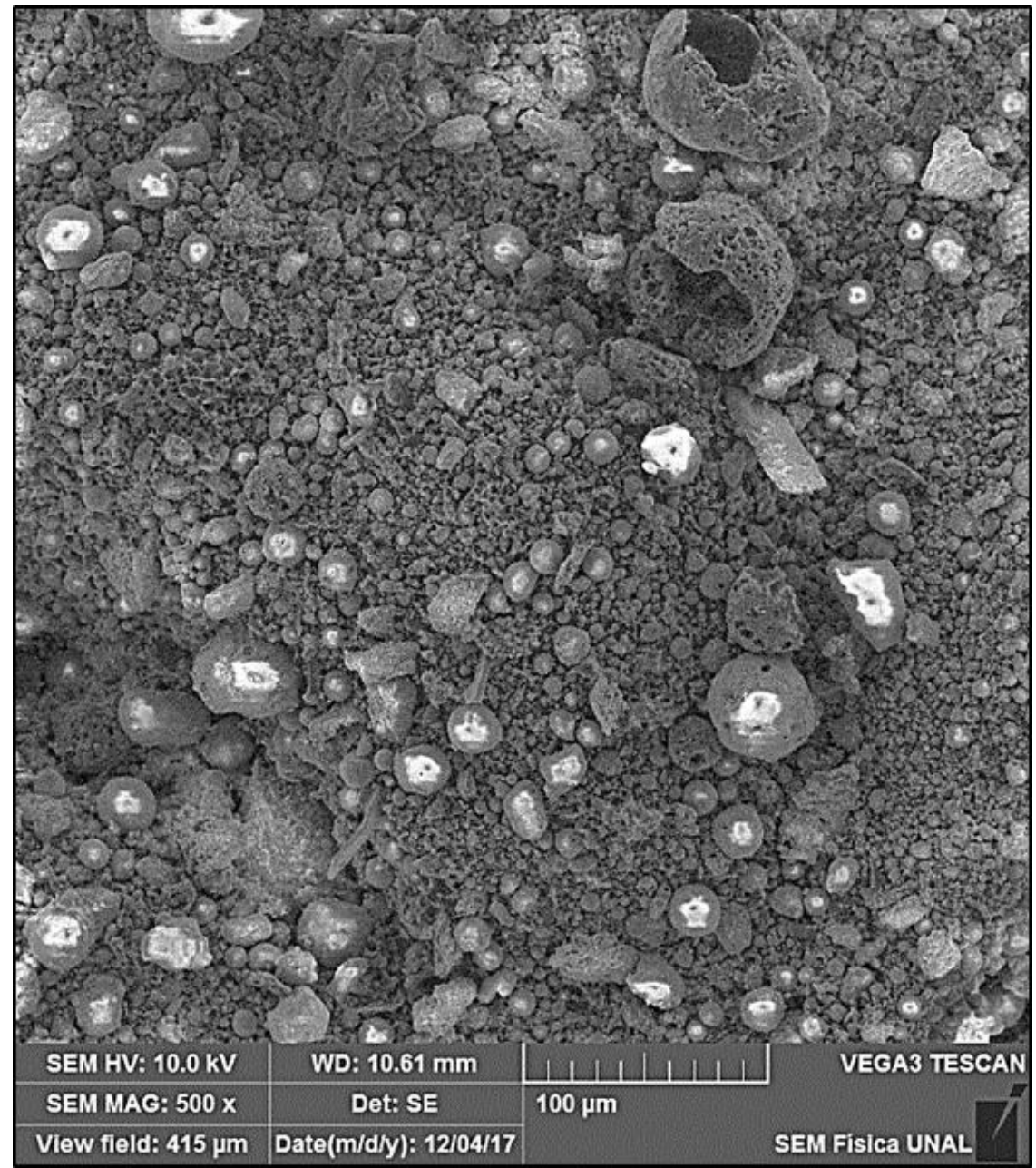

Fig. 4: Imagen SEM con detalle de morfología y diferentes componentes.
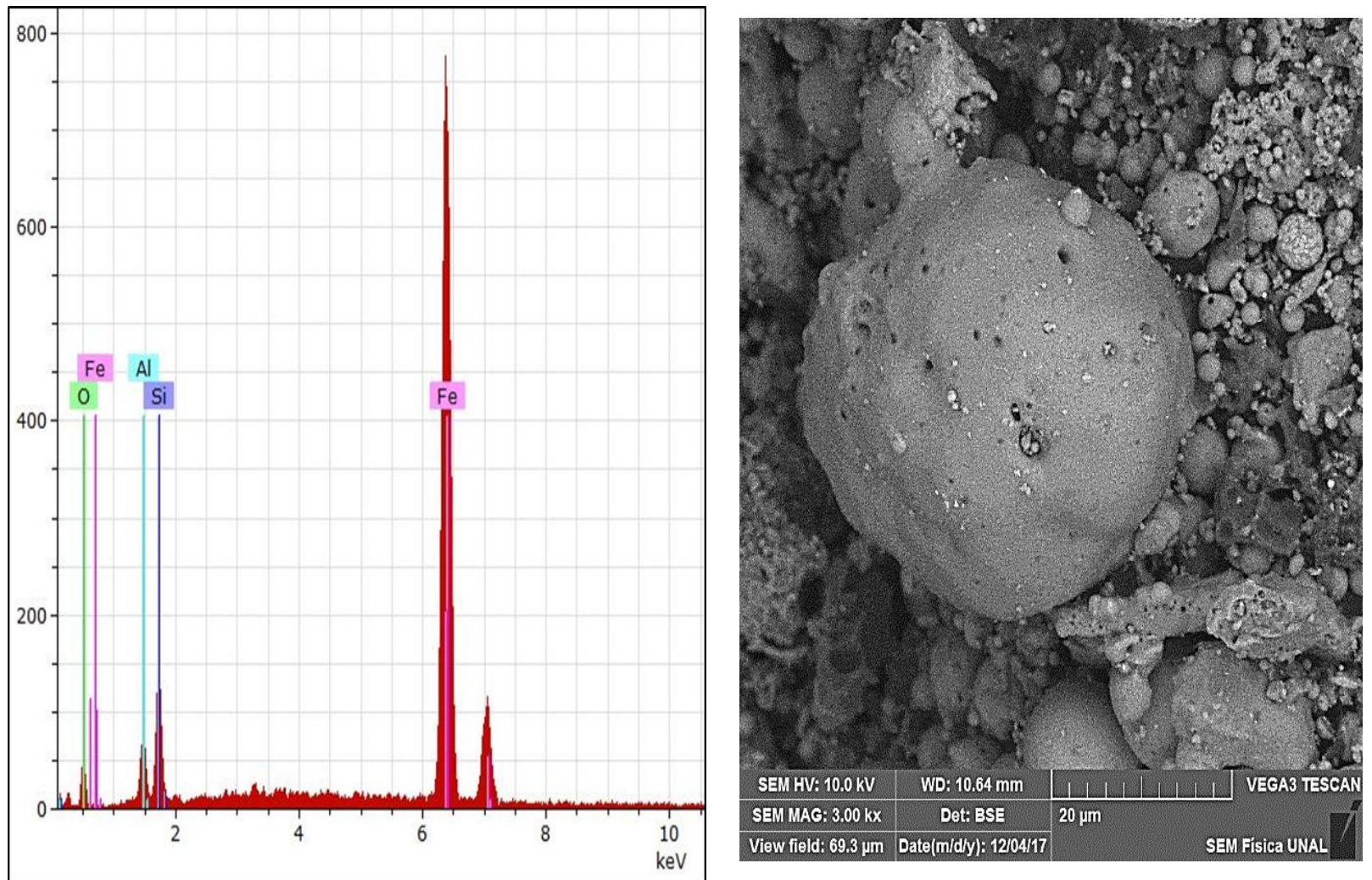

Fig. 5: Composición química y morfología de una partícula de ceniza volante. 


\section{Propiedades en estado fresco y endurecido}

La fluidez de los morteros de cemento fue de $107 \%$ consecuencia de la relación $A / C$ de 0,48 lo que muestra que los morteros son de consistencia plástica y tienen buena manejabilidad para obtener resistencias adecuadas en la elaboración de tabletas prefabricadas. Para los morteros de ceniza volante activada alcalinamente que obtuvieron mayor resistencia a la compresión (muestras GB6-24h y GB8-24h) la fluidez aumenta al disminuir la relación $\mathrm{AA} / \mathrm{CV}$. Las muestras reportan fluidez de $95 \%$ y $75 \%$ respectivamente, esto se debe a que experimentalmente se observó que a mayor cantidad en masa de $\mathrm{Na}_{2} \mathrm{SiO}_{3}$ las partículas de ceniza volante se conglomeran y no existe una homogeneización completa lo que produce una mezcla con difícil trabajabilidad. Al comparar ambos morteros se observa que la fluidez del mortero de cemento está relacionada directamente con la relación $\mathrm{A} / \mathrm{C}$, mientras que la fluidez del mortero de ceniza volante depende de la cantidad de $\mathrm{Na}_{2} \mathrm{SiO}_{3}$.

Las resistencias a la compresión de los morteros de ceniza volante se presentan en la Fig. 6, donde se observan los comportamientos de estas al variar concentraciones de $\mathrm{NaOH}$, relaciones AA/CV, proporciones $\mathrm{Na}_{2} \mathrm{SiO}_{3} / \mathrm{NaOH}$, edades y tiempos de curado. Cada fila de gráficas de arriba abajo corresponde en su orden a edades de $24 \mathrm{~h}$ a $80^{\circ} \mathrm{C}, 7,14$ y 28 días a temperatura ambiente y de izquierda a derecha (columnas) indican las molaridades de $\mathrm{NaOH}$ de 8,10 y $12 \mathrm{M}$.

En la Fig. 6 se evidencia que la mayor resistencia a la compresión se dio en la muestra GB6-24h siendo esta la mezcla óptima deseada para la elaboración de tabletas prefabricadas. Se observa que la concentración del $\mathrm{NaOH}$ desempeña un papel vital en el logro de la resistencia a la compresión. Esta aumenta significativamente cuando se utiliza una concentración de $\mathrm{NaOH}$ a $10 \mathrm{M}$, más allá de que se presenta una declinación en la resistencia cuando la concentración aumenta a $12 \mathrm{M}$ y disminuye a $8 \mathrm{M}$. La concentración de $\mathrm{NaOH}$ a $10 \mathrm{M}$ proporciona un ambiente altamente alcalino (aumento óptimo del $\mathrm{PH}$ ) y la cantidad de $\mathrm{Na}_{2} \mathrm{O}$ en el medio, favoreciendo la disolución de alúmina $(\mathrm{Al})$ y sílice $(\mathrm{Si})$ procedente de la ceniza, precursores principales de los compuestos que se producen en el proceso de hidratación del cemento geopolimérico aumentando su resistencia a la compresión (Lizarazo-Marriaga et al., 2015). Sin embargo, al aumentar la concentración de $\mathrm{NaOH}$ a $12 \mathrm{M}$ el proceso de geopolimerización es afectado por la cantidad excesiva de iones $\mathrm{OH}$ - arrojando resistencias bajas en comparación con las dos molaridades anteriores, lo cual no es recomendable para aplicaciones de concretos que demanden altas resistencias.

Por otro lado, el efecto de la proporción $\mathrm{Na}_{2} \mathrm{SiO}_{3} / \mathrm{NaOH}$ en la resistencia a la compresión de la muestra GB6$24 \mathrm{~h}$ tiene un aumento significativo cuando la proporción es de 1,50. Esto se puede atribuir a que la adecuada incorporación de $\mathrm{Na}_{2} \mathrm{SiO}_{3}$ en el sistema genera un aporte de sílice adicional $\left(\mathrm{SiO}_{2}\right)$ lo que conduce a formación de más enlaces Si-O-Si (Azevedo y Strecker, 2017; Lăzărescu et al., 2017). Cuando la proporción $\mathrm{Na}_{2} \mathrm{SiO}_{3} / \mathrm{NaOH}$ es de 2 y 2,50 la resistencia a la compresión disminuye porque el exceso de $\mathrm{Na}_{2} \mathrm{SiO}_{3}$ produce más iones de silicio $\left(\mathrm{Si}^{4+}\right)$ y de sodio $\left(\mathrm{Na}^{+}\right)$de los requeridos, lo que puede saturar la matriz con iones libres produciendo una inadecuada geopolimerización (Hadi et al., 2018). De igual modo, cuando el $\mathrm{Si}^{4+}$ y el $\mathrm{Na}^{+}$se reduce en el sistema la formación de la estructura geopolimérica se afecta negativamente, lo que genera disminución en la resistencia a la compresión (Hadi et al., 2018), tal como se evidencia en la relación $\mathrm{Na}_{2} \mathrm{SiO}_{3} / \mathrm{NaOH}$ de 1.

Las relaciones $A A / C V$ representan el peso total de las soluciones alcalinas $\left(\mathrm{Na}_{2} \mathrm{SiO}_{3} / \mathrm{NaOH}\right)$ sobre el peso de la ceniza volante. Los resultados mostraron que se obtuvo una mayor resistencia a la compresión cuando se utilizó relación AA/CV 0,45 tal como sucedió en la mezcla optima hallada (GB6-24h). Esto se puede atribuir a que al introducir la porción adecuada de activador alcalino a la ceniza volante el componente aluminosilicato de la ceniza es disuelto por la solución alcalina formando gel aluminosilicato, completando exitosamente la etapa de gelación y reorganización de los iones (Jiménez y Sánchez, 2007). Para relación AA/CV 0,40 la resistencia a la compresión se reduce por la cantidad insuficiente del activador alcalino que se requiere para disolver las partículas de ceniza volante, lo que puede causar la formación de un mortero con estructura no homogénea y partículas de ceniza volante sin reaccionar (Chi, 2015). Las relaciones AA/CV 0,50 y 0,60 tienen mejor trabajabilidad de la mezcla por la cantidad excesiva de activador alcalino que conduce a geopolímeros más porosos (Jiménez y Sánchez, 2007). En consecuencia, es probable que el aumento de activador alcalino inhiba el proceso de geopolimerización y repercuta en la disminución de la resistencia a la compresión (Hadi et al., 2018).

La Fig. 7 muestra las resistencias a la compresión de la mezcla óptima de ceniza volante (GB6) curada a diferentes edades comparadas con las resistencias a la compresión obtenidas por el cemento Portland. El curado para la ceniza volante a la edad de 1 día fue a $80^{\circ} \mathrm{C}$, para 7,14 y 28 días las muestras se curaron a temperatura ambiente. Los resultados indican que los morteros de cemento Portland curados a temperatura ambiente presentan mejores resistencias a la compresión que los del geopolímero. Cuando la ceniza volante activada alcalinamente se cura a temperatura ambiente la reacción de geopolimerización es más lenta, 
(Palomo et al., 2004). En consecuencia, las resistencias óptimas se obtienen en tiempos más prolongados en comparación con el cemento Portland. La resistencia adquirida por el cemento a 7 días (13,55 MPa) la ceniza volante la alcanza a los 28 días (16,02 MPa), para este caso el curado a temperatura ambiente para ceniza volante no es favorable en la industria de prefabricados.
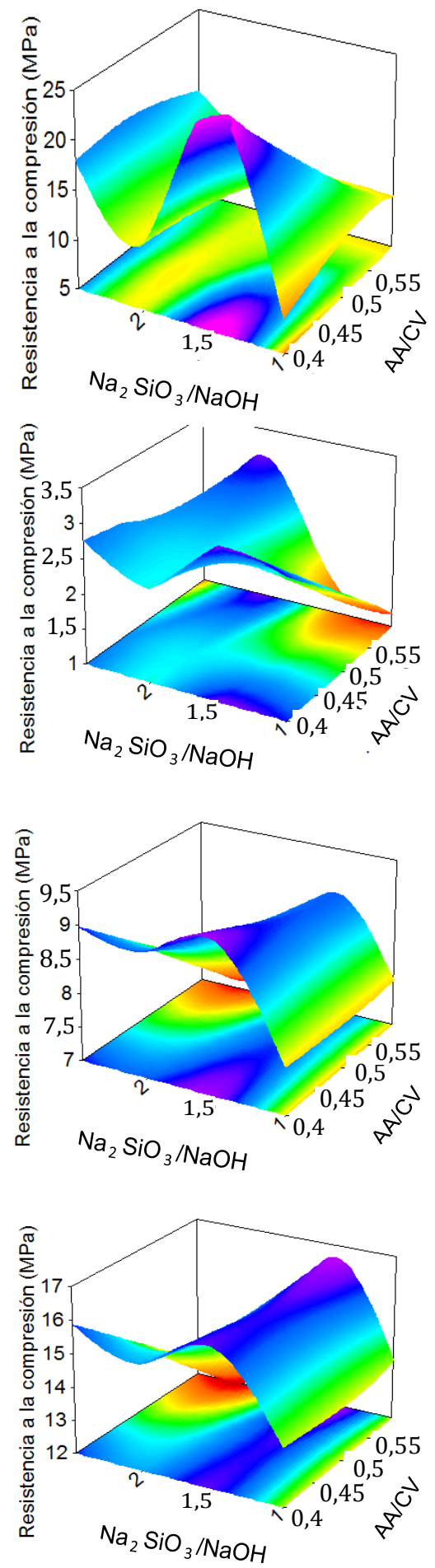
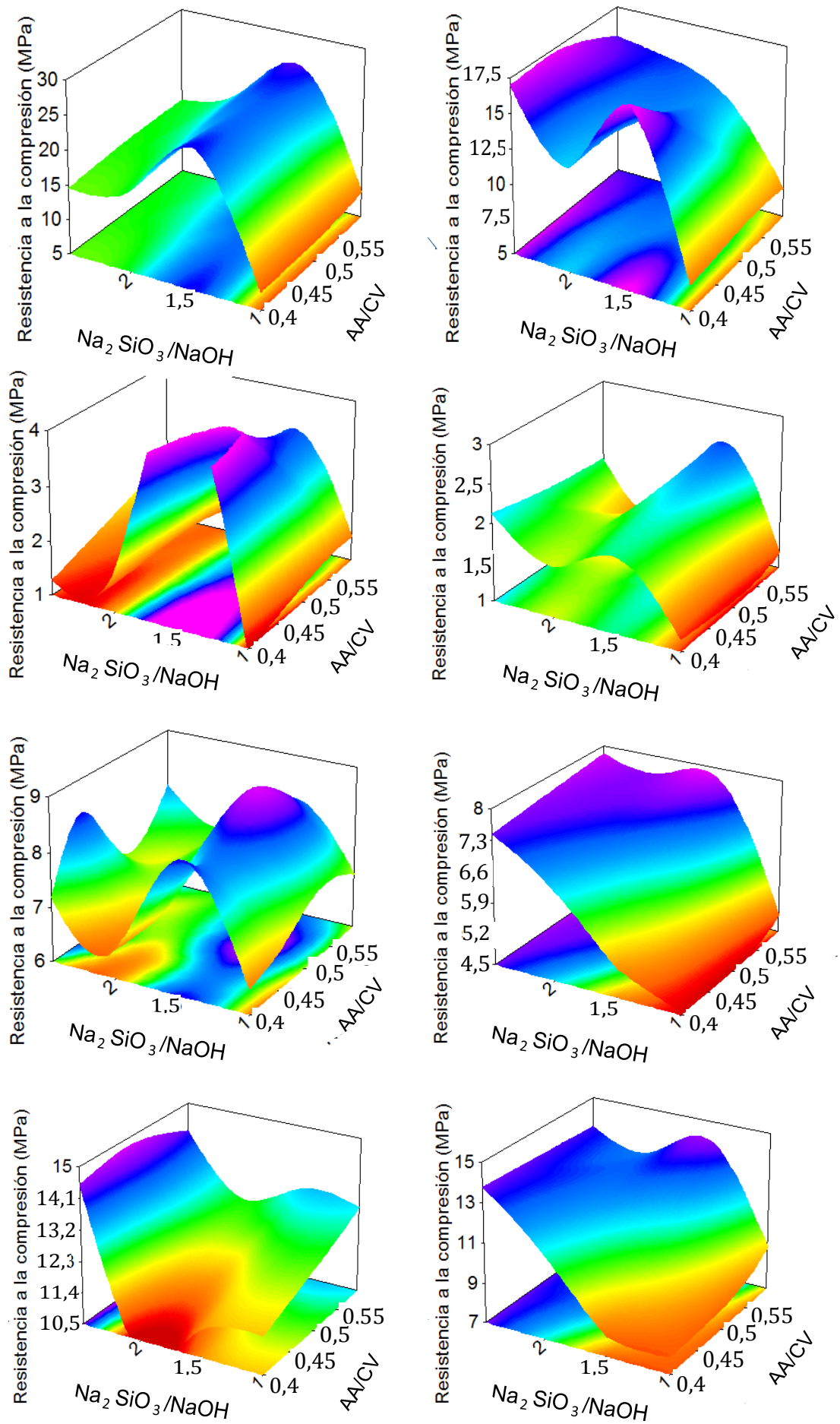

Fig. 6: Resistencias a la compresión de morteros de ceniza volante activada alcalinamente a diferentes edades de curado 


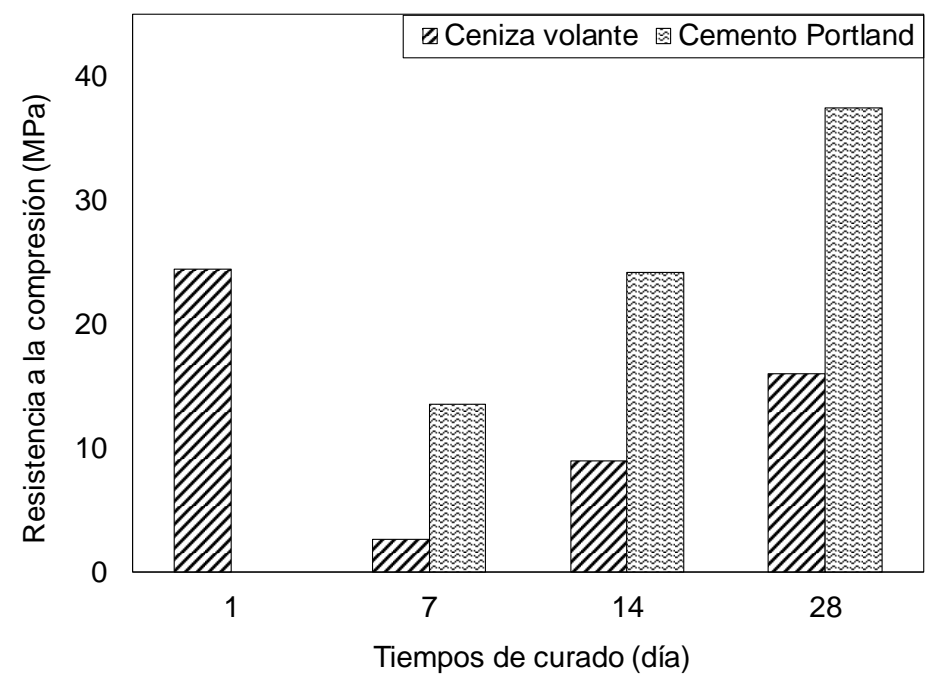

Fig. 7: Resistencias a la compresión de morteros de ceniza volante activada alcalinamente y cemento Portland a diferentes tiempos de curado.

Sin embargo, el mortero de ceniza volante curado a $24 \mathrm{~h}$ a temperatura $\left(80^{\circ} \mathrm{C}\right)$ obtiene la resistencia mínima para tabletas prefabricadas convencionales según la (NTC 4992, 2004). El aumento de temperatura incrementa el contenido de sílice, de modo que se altera la relación Si/Al del gel aluminosilicato alcalino formado en la reacción geopolimérica, lo que incide directamente en la ganancia de resistencia (Görhan y Kürklü, 2014). Es decir, el mortero de ceniza volante adquiere resistencia de $(24,46 \mathrm{MPa})$ en $24 \mathrm{~h}$ mientras que el mortero de cemento tarda 14 días. No obstante, las resistencias a la comprensión continúan aumentando de forma más lenta a lo largo del tiempo como sucede con los morteros de cemento Portland (Jiménez y Sánchez, 2007).

Aunque el hormigón de cemento Portland también se puede curar con temperatura inducida para obtener resistencias óptimas en corto tiempo, esto puede generar efectos adversos. La rápida hidratación del cemento produce desarrollo mecánico inicial favorable, pero retarda la hidratación posterior de las partículas que inicialmente fueron hidratadas, produciendo desarrollo negativo de las resistencias mecánicas a medio y largo plazo (Jiménez y Sánchez, 2007). Por tanto el curado en condiciones de humedad para el cemento portland garantiza que la mayoría de partículas reaccionen exitosamente producto de alto contenido de humedad y temperatura en las muestras, traduciéndose esto en buena resistencia a la compresión y durabilidad a largo plazo (Lizarazo et al., 2016). Por otro lado, la utilización de acelerantes también puede ayudar en la obtención de resistencias a corto plazo para el cemento Portland, lo que puede aumentar los costos de producción. Ahora bien, la implementación de acelerantes en el cemento Portland no hace parte de la presente investigación de manera que no se incluyó en los morteros estudiados.

En términos generales, la implementación de la ceniza volante en la industria de prefabricados podría competir directamente con el cemento Portland por la capacidad de obtener buenas resistencias en un tiempo más corto. Finalmente, al realizar el ensayo módulo de rotura para tres tabletas prefabricadas de mortero de ceniza volante de la mezcla óptima (GB6-24h) se obtiene un valor promedio de 7,45 MPa. Al igual que ocurre con la resistencia a la compresión las tabletas prefabricadas de ceniza volante cumplen con el valor mínimo establecido por la (NTC 4992, 2004).

\section{Huella de carbono}

Para la cuantificación de $\mathrm{CO} 2$ eq del alcance 1 en la fabricación de tabletas prefabricadas de mortero de cemento se tuvo en cuenta la recepción del cemento y la arena por parte de un depósito cercano a la Universidad de La Salle ubicado en Bogotá Colombia. En las tabletas prefabricadas de mortero de ceniza volante se consideró el transporte asociado al traslado de la ceniza volante desde la termoeléctrica Termozipa hasta la Universidad de La Salle. Para calcular la cantidad de Kg CO2 eq de los alcances 1 y 2 para mil (1000) kilogramos de tabletas prefabricadas de cada material se usaron las ecuaciones. (1) y (2) teniendo en cuenta los tipos de combustibles, poderes caloríficos y factores de emisión establecidos para los combustibles colombianos (FECOC) (Upme, 2003).

En las Tablas 3 y 4 se presentan los resultados de las emisiones de $\mathrm{CO} 2$ eq obtenidos en la producción de un lote de mil (1000) kilogramos de tabletas prefabricadas de cada material. En el cálculo de la huella de carbono del alcance dos de las tabletas prefabricadas de mortero de cemento se tuvieron en cuenta las 
emisiones desde la extracción de materias primas del cemento, datos obtenidos de la planta visitada. Para el cálculo de la huella de carbono del alcance dos en las tabletas prefabricadas de ceniza volante no se tuvo en cuenta la extracción de materias primas de los activadores alcalinos debido a que varía el valor dependiendo del lugar de extracción, distancias y maquinaria utilizada.

Los resultados obtenidos indican que las tabletas prefabricadas de ceniza volante tienen una huella de carbono menor con respecto a las tabletas prefabricadas de cemento Portland como se puede evidenciar en la Fig. 8. En el alcance 1 a pesar de que los procesos son muy similares las tabletas prefabricadas de ceniza volante obtienen un valor más alto de $\mathrm{CO} 2$ eq por la necesidad de utilizar el horno para curar las tabletas prefabricadas. Aunque en el presente estudio se realizó curado a temperatura ambiente en diferentes tiempos no se obtuvieron resistencias óptimas. Sin embargo, (Kiran Kumar y Gopala Krishna Sastry, 2017) afirman que la ceniza volante puede obtener buenas resistencias a la compresión a temperatura ambiente adicionando materiales que aumenten la cantidad de sílice a la mezcla. Por otro lado, el transporte utilizado para adquirir la ceniza volante aumenta las emisiones de $\mathrm{CO} 2$ eq porque este material solo se puede obtener en el lugar de origen, mientras que el cemento Portland puede ser adquirido en cualquier depósito de materiales.

Según la Fig. 8 las emisiones del alcance 2 de las tabletas prefabricadas de ceniza volante activada alcalinamente son notablemente más bajas que las fabricadas en cemento Portland. Esto se debe a que la materia prima (ceniza volante) es un residuo aprovechado de la producción de energía. En consecuencia, al igual que (Chan et al., 2016) las emisiones de CO2 de la termoeléctrica no se toman en cuenta por que el objetivo de la termoeléctrica es producir energía y no ceniza volante. Por el contrario en la producción de cemento Portland se generan emisiones por combustión y consumo energético principalmente en los procesos de molienda, precalentamiento, calcinación y enfriamiento, no obstante estas emisiones pueden ser reducidas implementando tecnología con eficiencia energética (Osorio et al., 2012). Por tanto, se comprueba que la incorporación de ceniza volante en la industria de prefabricados es viable ambientalmente en términos de huella de carbón generando una disminución en emisiones totales de $\mathrm{CO} 2$ del 55\% en comparación con las tabletas prefabricadas de cemento Portland.

Tabla 3: Energía y emisiones de $\mathrm{CO}_{2}$ para tabletas prefabricadas de mortero de cemento Portland.

\begin{tabular}{|c|c|c|c|c|}
\hline & $\begin{array}{l}\text { Energía } \\
(\mathrm{Wh} / \mathrm{Kg})\end{array}$ & $\begin{array}{c}\text { Cantidad de } \\
\text { combustible }(\mathrm{Kg})\end{array}$ & $\begin{array}{c}\text { Tipo de } \\
\text { combustible }\end{array}$ & $\begin{array}{l}\text { Emisión } \\
\left(\mathrm{Kg} \mathrm{CO}_{2}-e q\right.\end{array}$ \\
\hline \multicolumn{5}{|l|}{ Alcance 2} \\
\hline Extracción de materias primas & & 0,13 & Diésel & 0,40 \\
\hline Trituración de materia prima & 0,93 & & & 0,35 \\
\hline Prehomogenización & 1,50 & & & 0,56 \\
\hline Molienda de materia prima & 28,60 & & & 10,70 \\
\hline Homogeneización (mezcla en seco) & 0,95 & & & 0,36 \\
\hline $\begin{array}{l}\text { Precalentamiento, calcinación y } \\
\text { enfriamiento }\end{array}$ & 100 & 98 & Coque & 324,05 \\
\hline Molienda del cemento & 39,70 & & & 14,85 \\
\hline \multirow[t]{2}{*}{ Envase y embarque del producto } & 15 & & & 5,61 \\
\hline & & Subtotal & & 356,88 \\
\hline \multicolumn{5}{|l|}{ Alcance 1} \\
\hline $\begin{array}{l}\text { Transporte de insumos del depósito } \\
\text { a laboratorios U. Salle }\end{array}$ & & 0,36 & Gasolina & 1,05 \\
\hline $\begin{array}{l}\text { Mezcla de cemento, agua y } \\
\text { agregados }\end{array}$ & 2,55 & & & 0,95 \\
\hline \multirow{2}{*}{$\begin{array}{l}\text { Moldeado y vibro-comprimido de } \\
\text { tableta }\end{array}$} & 1,39 & & & 0,52 \\
\hline & & Subtotal & & 2,52 \\
\hline & Total & & & 360,40 \\
\hline
\end{tabular}


Tabla 4: Energía y emisiones de $\mathrm{CO}_{2}$ para tabletas prefabricadas de mortero de ceniza volante activada alcalinamente.

\begin{tabular}{|c|c|c|c|c|}
\hline & $\begin{array}{l}\text { Energía } \\
(\mathrm{Wh} / \mathrm{Kg})\end{array}$ & $\begin{array}{c}\text { Cantidad de } \\
\text { combustible (Kg) }\end{array}$ & $\begin{array}{c}\text { Tipo de } \\
\text { combustible }\end{array}$ & $\begin{array}{l}\text { Emisión } \\
\left(\mathrm{Kg} \mathrm{CO} \mathrm{CO}_{2} \text { eq) }\right.\end{array}$ \\
\hline \multicolumn{5}{|l|}{ Alcance 2} \\
\hline $\begin{array}{l}\text { Fundición de arena de sílice y } \\
\text { carbonato de sodio }\left(\mathrm{Na}_{2} \mathrm{SiO}_{3}\right)\end{array}$ & & 30,75 & Diésel & 97,50 \\
\hline \multirow[t]{2}{*}{ Electrólisis de salmuera $(\mathrm{NaOH})$} & 75 & & & 28,05 \\
\hline & & Subtotal & & 125,55 \\
\hline \multicolumn{5}{|l|}{ Alcance 1} \\
\hline $\begin{array}{l}\text { Transporte de ceniza volante a } \\
\text { laboratorios U.Salle }\end{array}$ & & 2,31 & Gasolina & 6,79 \\
\hline Mezcla de cemento, agua y arena & 2,55 & & & 0,95 \\
\hline Moldeado y vibro-comprimido de tableta & 1,39 & & & 0,52 \\
\hline \multirow[t]{3}{*}{ Curado tableta $\left(80^{\circ} \mathrm{C}\right)$} & 76,80 & & & 28,72 \\
\hline & & Subtotal & & 36,98 \\
\hline & Total & & & 162,53 \\
\hline
\end{tabular}

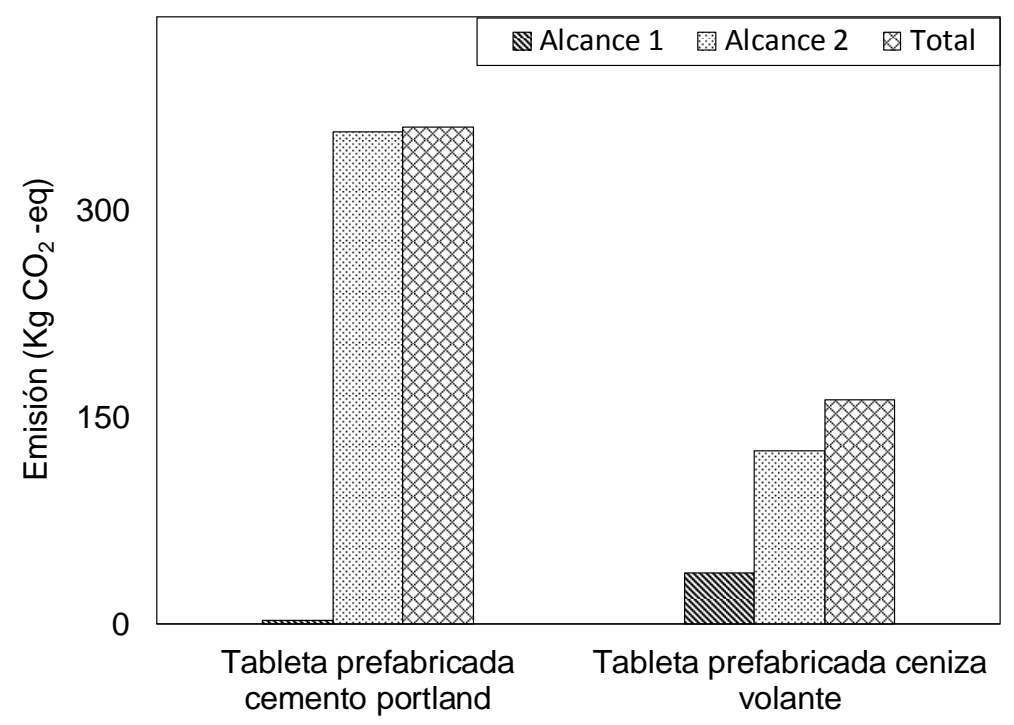

\section{CONCLUSIONES}

Fig. 8: Cuantificación total de emisiones de $\mathrm{CO}_{2}$-eq.

De acuerdo a los resultados se extraen las siguientes conclusiones principales: 1) este estudio demostró que el uso de ceniza volante generada por la combustión de carbón colombiano es apta para ser activada alcalinamente y obtener resistencias a la compresión y flexión similares a las del cemento Portland en tabletas prefabricadas; 2) los resultados mecánicos indicaron que al igual que los morteros de cemento Portland, las buenas propiedades mecánicas de los morteros de ceniza volante activada alcalinamente están influenciadas por una adecuada dosificación de la mezcla y condiciones de curado, por esta razón la muestra GB6-24h fue la mezcla óptima deseada; 3) la ceniza volante activada alcalinamente es competitiva en la industria de tabletas prefabricadas cuando es curada a temperatura inducida $\left(80^{\circ} \mathrm{C}\right)$ porque se obtienen buenas resistencias a la compresión y módulo de rotura a edades cortas (1dia); 4) el cálculo de la huella de carbono demostró que el uso de ceniza volante activada alcalinamente en tabletas prefabricadas reduce el $55 \%$ de emisiones de $\mathrm{CO}_{2}$ eq en comparación con el cemento Portland. 


\section{REFERENCIAS}

Association World Coal, UK (2018)

ASTM C33, Standard Specification for Concrete Aggregates, Pensilvania, EUA (2018)

ASTM C109M, Standard Test Method for Compressive Strength of Hydraulic Cement Mortars, Pensilvania, EUA (2016)

ASTM C150, Standard Specification for Portland Cement, Pensilvania, EUA (2014)

ASTM C348-14, Standard Test Method for Flexural Strength of Hydraulic-Cement Mortars, Pensilvania, EUA (2014)

ASTM C618-12, Standard Specification for Coal Fly Ash or Calcined Natural Pozzolan for Use in Concrete, Pensilvania, EUA (2014)

Azevedo, A.G.D.S. y K. Strecker, Brazilian Fly Ash Based Inorganic Polymers Production Using Different Alkali Activator Solutions, doi:10.1016/j.ceramint.2017.04.044, Ceramics International, 43(12), 9012-9018 (2017)

Bautista-Ruiz, W.A., M. Díaz y S.A. Martínez-Ovalle, Caracterización de las Cenizas Volantes de una Planta Termoeléctrica para su Posible Uso como Aditivo en la Fabricación de Cemento, doi:10.19053/20278306.v8.n1.2017.7374, Revista de Investigación, Desarrollo e Innovación, 8(1), 135-146 (2017)

Chan, C.C.S., D. Thorpe y M. Islam, An Evaluation Carbon Footprint in Fly Ash Based Geopolymer Cement and Ordinary Portland Cement Manufacture, doi: 10.1109/IEEM.2015.7385647, IEEE International Conference on Industrial Engineering and Engineering Management, 254-259 (2016)

Chi, M., Effects of Modulus Ratio and Dosage of Alkali-Activated Solution on the Properties and Micro-Structural Characteristics of Alkali-Activated Fly Ash Mortars, doi:10.1016/j.conbuildmat.2015.09.029, Construction and Building Materials, 99, 128-136 (2015)

Chithiraputhi, S. y N. Neithalath, Isothermal Reaction Kinetics and Temperature Dependence of Alkali Activation of Slag, Fly Ash and Their Blends, doi: 10.1016/j.conbuildmat.2013.03.061, Construction and Building Materials, 45, $233-242$ (2013)

DANE, Estadísticas de Cemento Gris- Ecg., Colombia (2018a)

DANE, Producto Interno Bruto Primer Trimestre 2018, Colombia (2018b)

European Comission DG Environmental, Product Carbon Footprinting - a Study on Methodologies and Initiatives, UE (2010)

Espíndola, C. y J.O. Valderrama, Huella Del Carbono. Parte 1: Conceptos, Métodos de Estimación y Complejidades Metodológicas, doi: 10.4067/S0718-07642012000100017, Información Tecnológica, 23(1), 163-176 (2012)

Görhan, G. y G. Kürklü, The Influence of the Naoh Solution on the Properties of the Fly Ash-Based Geopolymer Mortar Cured at Different Temperatures, doi: 10.1016/j.compositesb.2013.10.082, Composites Part B: Engineering, 58, 371-377 (2014)

Hadi, M.N.S., M. Al-Azzawi y T. Yu, Effects of Fly Ash Characteristics and Alkaline Activator Components on Compressive Strength of Fly Ash-Based Geopolymer Mortar; doi: 10.1016/j.conbuildmat.2018.04.092, Construction and Building Materials, 175, 41-54 (2018)

Hoyos-Montilla, A.A., F. Puertas y J.I. Tobón, Microcalorimetric Study of the Effect of Calcium Hydroxide and Temperature on the Alkaline Activation of Coal Fly Ash, doi: 10.1007/s10973-017-6715-4, Journal of Thermal Analysis and Calorimetry, 131(3), 2395-2410 (2018)

Jiménez, A.F. y A. Palomo, Factores Que Afectan Al Desarrollo Inicial De Resistencias a Compresión En Hormigones De Ceniza Volante Activados Alcalinamente (Sin Opc), doi: 10.3989/mc.2007.v57.i287.53, Materiales de Construcción, 57 (287), 7-22 (2007)

Khan, M.Z.N., F.u.A. Shaikh, Y. Hao y H. Hao, Synthesis of High Strength Ambient Cured Geopolymer Composite by Using Low Calcium Fly Ash, doi: 10.1016/j.conbuildmat.2016.08.097, Construction and Building Materials, 125, $809-820$ (2016)

Kiran-Kumar, N.L.N. y K.V.S. Gopala Krishna Sastry, Effects of Nano Silica on the Strengths of Geopolymer Concrete Cured at Ambient Temperature, doi: 10.1016/j.conbuildmat.2015.10.044, International Journal of Civil Engineering and Technology, 8(8), 437-444 (2017)

Lizarazo, J.M., A. Salas y D.A. Escobar, Efectos del Curado en las Propiedades de Mezclas de Concreto con Altos Contenidos de Escoria de Hierro, doi: 10.4067/S0718-07642016000600017, Información Tecnológica, 27(6), 163-174 (2016)

Lizarazo-Marriaga, J., F. García y C. Higuera, Chemical Electromutagenic Activation of the Cementitious Properties of Fly Ash, Revista Latinoamericana de Metalurgia y Materiales, ISSN: 0255-6952 35(2), 305-314 (2015)

Lǎzărescu, A.V., H. Szilagyi, C. Baeră y A. Ioani, The Effect of Alkaline Activator Ratio on the Compressive Strength of Fly Ash-Based Geopolymer Paste, doi:10.1088/1757-899X/209/1/012064, IOP Conference Series: Materials Science and Engineering, 209(1), (2017)

Mejía, J.M., E.D. Rodríguez y R. Mejía de Gutiérrez, Utilización Potencial De Una Ceniza Volante de Baja Calidad Como Fuente de Aluminosilicatos en la Producción De Geopolímeros, doi: 10.11144/Javeriana.IYU18-2.upcv, Ingeniería y Universidad, 18(2), 309-327 (2014)

NTC 4992, Loseta de Concreto para Pavimentos, Bogotá D.C., Colombia (2004) 
Osorio A.M., J.M. Marín y G. Restrepo, Diseño y Evaluación Energética de dos Circuitos de Molienda y Clasificación para un Clincker de Cemento a Escala Piloto, doi: 10.4067/S0718-07642013000200011, Información Tecnológica, 24(2), 99108 (2013)

Palomo, A., S. Alonso y otros tres autores, Alkaline Activation of Fly Ashes: Nmr Study of the Reaction Products, doi: 10.1111/j.1551-2916.2004.01141.x, Journal of the American Ceramic Society, 87(6), 1141-1145 (2004)

PAS 2050, Norma Publicly Available Specification, Londres, Reino Unido (2011)

Rieger, D., L. Kullová y otros tres autores, Mechanically Activated Fly Ash as a High Performance Binder for Civil Engineering, doi: 10.1088/1742-6596/790/1/012030, Journal of Physics: Conference Series, 790(1), (2017)

Saloma, A. Saggaff, Hanafiah y A. Mawarni, Geopolymer Mortar with Fly Ash, doi:10.1051/matecconf/20167801026, MATEC Web of Conferences, 78 (2016)

Sánchez-Soloaga, I., A. Oshiro y M. Positieri, Contribución de la Reducción de La Huella Ecológica Del Hormigón. Avances en Energías Renovables y Medio Ambiente, ISSN: 0329-5184, 16, (2012)

Singh, G.V.P.B. y K.V.L. Subramaniam, Evaluation of Sodium Content and Sodium Hydroxide Molarity on Compressive Strength of Alkali Activated Low-Calcium Fly Ash, doi:10.1016/j.cemconcomp.2017.05.001, Cement and Concrete Composites, 81, 122-132 (2017a)

Singh, G.V.P.B. y K.V.L. Subramaniam, Role of Total Reactive Oxide Ratios on Strength Development in Activated Fly Ash, doi:10.1051/matecconf/201712002014, International Conference on Advances in Sustainable Construction Materials \& Civil Engineering Systems, 120, (2017b)

UNE-EN 15804, Sostenibilidad en la Construcción. Declaraciones Ambientales de Producto. Reglas de Categoría de Producto Básicas para Productos de Construcción, Madrid, España (2014)

UNE 127757,2016, Reglas de Categoría de Producto para la Obtención de Declaraciones Ambientales de Productos Prefabricados de Hormigón, Madrid, España (2018)

UPME, Factores de Emisión de Los Combustibles Colombianos, Academia Colombiana de Ciencias Exactas Físicas y Naturales, Colombia (2003a)

UPME, Plan Energético Nacional Colombia, Ideario Energético 2050, Colombia (2015b)

Velásquez-Vallejo, L.F., J.F. De La Cruz Morales, J.F. Sánchez Morales y M.A Marín Laverde, Remoción De Carbón Inquemado de Las Cenizas Volantes Producidas en el Proceso de Combustión de Carbón, Revista Energética, ISSN: 2357-612X, (38), 107-112 (2007)

Yousefi Oderji, S., B. Chen y S.T.A. Jaffar, Effects of Relative Humidity on the Properties of Fly Ash-Based Geopolymers, doi: 10.1016/j.conbuildmat.2017.07.115, Construction and Building Materials, 153, 268-273 (2017) 
